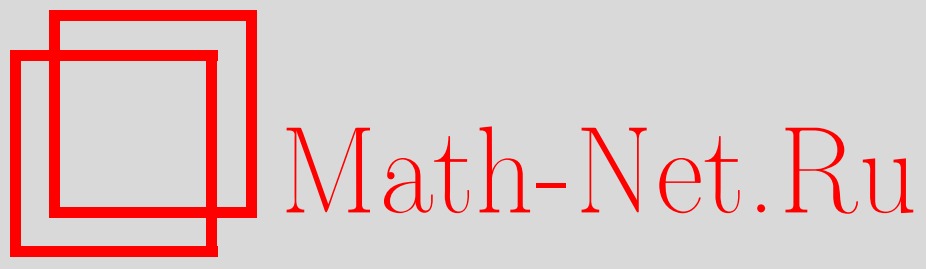

С. В. Шадрин, Дополнение к бифуркационной диаграмме тригонометрических полиномов, Функи. анализ и его прил., 2001, том 35, выпуск 1, 93-95

DOI: https://doi.org/10.4213/faa239

Использование Общероссийского математического портала MathNet.Ru подразумевает, что вы прочитали и согласны с пользовательским соглашением

http://www . mathnet.ru/rus/agreement

Параметры загрузки:

IP : 18.208 .226 .222

26 апреля 2023 г., 05:50:17

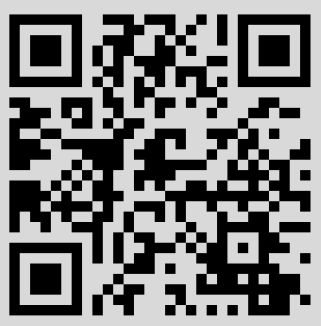




\title{
ЛИТЕРАТУРА
}

1. Математическая энциклопедия. Т. 2, Советская энциклопедия, M, 1979. 2. Szego G. In: Festskrift Marcel Riesz (Lund. 1952), pp. 228-238. 3. Гренандер У., Сегё Г. Теплицевы формы и их приложения. ИЛ, М., 1961. 4. Линник И.Ю. Изв. АН СССР, сер. матем., 39, №6, 1393-1403 (1975). 5. Widom H. Trans. Amer. Math. Soc., 94, No. 1, 170-180 (1960). 6. Böttcher A., Silbermann B. Invertibility and Asymptotics of Toeplitz Matrices. Akademie-Verlag, Berlin, 1983.

Ростовский госуниверситет

Поступило в редакцию 18 мая 1999 г.

УДК 517.3

\section{Дополнение к бифуркационной диаграмме тригонометрических полиномов}

\author{
(c) 2001. С. В. ШАДРИн
}

1. Мы рассматриваем пространство вещественных тригонометрических полиномов $F=\left\{f(t)=\cos n t+\sum_{j=1}^{n-1}\left(A_{j} \cos j t+B_{j} \sin j t\right) \mid A_{j}, B_{j} \in \mathbb{R}\right\}$, определенных на окружности $\left(f: S^{1}=\mathbb{R} / 2 \pi \mathbb{Z} \rightarrow \mathbb{R}\right)$. Бифуркациионной диаграммой $\Sigma_{1} \subset F$ называется множество неморсовских полиномов.

Комплексной формой полинома $f \in F$ мы называем мероморфную функцию $g(z): \overline{\mathbb{C}} \rightarrow \overline{\mathbb{C}}$, получаемую из $f$ заменой $z=e^{i t}$. Обозначим через $\Sigma_{2}$ множество полиномов $f \in F$, комплексные формы которых имеют вещественные непростые критические значения.

Очевидно, что $\Sigma_{1}$ и $\Sigma_{2}-$ гиперповерхности в $F$, причем $\Sigma_{1} \subset \Sigma_{2}$. Статья посвящена описанию дополнений $F \backslash \Sigma_{1}$ и $F \backslash \Sigma_{2}$. Ранее аналогичные вопросы рассматривались в [1] для обычных полиномов и в $[2,3]$ для тригонометрических полиномов с $2 n$ критическими точками на окружности.

2. Функции $f_{1}, f_{2}: S^{1} \rightarrow \mathbb{R}$ называются топологически эквивалентныли, если существуют сохраняющие ориентацию гомеоморфизмы $\alpha: S^{1} \rightarrow S^{1}, \beta: \mathbb{R} \rightarrow \mathbb{R}$, такие, что $f_{1} \alpha=\beta f_{2}$. Очевидно, что если $f \in F$ и $f^{\prime} \in F-$ топологически неэквивалентные морсовские полиномы, то они лежат в разных компонентах дополнения $F \backslash \Sigma_{1}$.

Рассмотрим морсовскую функцию $f_{0}: S^{1} \rightarrow \mathbb{R}$. Обозначим через $\left[f_{0}\right]$ подмножество в $F$, состоящее из всех морсовских полиномов, топологически эквивалентных $f_{0}$. Пусть число критических точек функции $f_{0}$ на окружности равно $2 k$. Очевидно, что если $k>n$, то $\left[f_{0}\right]$ пусто. Поэтому в дальнейшем мы во всех рассуждениях будем предполагать, что $k \leqslant n$.

ТЕОремА 1. Для любой морсовской функции $f_{0}$, такой, что $k \leqslant n$, множество $\left[f_{0}\right]$ непусто. Кроме того, если $k<n$, то $\left[f_{0}\right]-$ связное подмногообразие в $F$. Если же $k=n$, то $\left[f_{0}\right]-$ подмногообразие в $F$, гомотопически эквивалентное $n$ точкам.

Эта теорема дает описание всех компонент дополнения $F \backslash \Sigma_{1}$.

3. Скобочным символом называется синтаксически правильная последовательность из $2 k$ пар квадратных скобок, идущих подряд, и $n-k$ пар круглых скобок, 
вписанных в квадратные. Для примера перечислим все скобочные символы для $n=3, k=1:[(())][],[()()][],[()][()],[][(())],[][()()]$. В [1] доказано, что при фиксированных $n$ и $k$ число всех скобочных символов равно $\left(\begin{array}{c}2 n-1 \\ n-k\end{array}\right)-\left(\begin{array}{c}2 n-1 \\ n-k-1\end{array}\right)$.

Каждому полиному $f \in\left[f_{0}\right] \backslash \Sigma_{2}$ мы поставим в соответствие скобочный символ. Для этого рассмотрим комплексную форму $g$ полинома $f \in F$. Очевидно, что $g(z)=\frac{1}{2}\left(z+z^{-1}\right)+\sum_{j=1}^{n-1}\left(C_{j} z^{j}+\bar{C}_{j} z^{-j}\right)$.

Опишем подмножество единичного круга, на котором $g$ принимает вещественные значения: это единичная окружность, $2 k$ линий $\lambda_{i}$, соединяющих нуль с критическими точками на единичной окружности и $n-k$ окружностей $\omega_{m}$, содержащих точку нуль. Иных точек пересечения, кроме явно указанных, перечисленные линии не имеют $\left(\lambda_{j} \cap \lambda_{i}=\lambda_{i} \cap \omega_{m}=\omega_{m} \cap \omega_{l}=\{0\}, 1 \leqslant i, j \leqslant 2 k\right.$, $1 \leqslant m, l \leqslant n-k)$.

Сделаем обход вокруг нуля в положительном направлении, стартуя с линии $\lambda_{1}$, соединяющей нуль с глобальным минимумом функции $g$ на единичной окружности. При пересечении с прообразом вещественных чисел мы будем выставлять скобки, руководствуясь следующими правилами: 1) в начале ставим «[», в конце - «]»; 2) при пересечении линии $\lambda_{i}, 2 \leqslant i \leqslant 2 k$, мы ставим «][»; 3) при пересечении окружности $\omega_{m}, 1 \leqslant m \leqslant n-k$, в первый раз мы ставим «(», во второй раз «)». Естественно, обход предполагается таким, что каждую линию $\lambda_{i}$ мы пересечем один раз, а каждую окружность $\omega_{m}-$ два раза.

Очевидно, что полученная последовательность - скобочный символ. Мы будем называть его скобочным символом полинома $f$. Очевидно, что полиномы с различными скобочными символами лежат в разных компонентах дополнения $\left[f_{0}\right] \backslash \Sigma_{2}$.

Обозначим через $\left[f_{0}, \mathrm{smb}\right]$ подмножество в $\left[f_{0}\right] \backslash \Sigma_{2}$, состоящее из всех полиномов, скобочный символ которых равен smb.

ТЕорема 2. Для Каждого скобочного символа smb множество $\left[f_{0}, \mathrm{smb}\right]$ неnyсто. Более того, $\left[f_{0}, \mathrm{smb}\right]-$ подмногообразие в $\left[f_{0}\right]$, гомотопически эквивалентное п точкам.

Эта теорема дает полное описание всех компонент дополнения $F \backslash \Sigma_{2}$.

4. Изложим схему доказательства теоремы 2 . На пространстве $F$ действует группа $\mathbb{Z}_{n}$, порожденная отображением $\varphi: f(t) \mapsto f(t+2 \pi / n)$. Очевидно, что $\varphi\left(\Sigma_{i}\right)=\Sigma_{i}, i=1,2, \varphi\left(\left[f_{0}\right]\right)=\left[f_{0}\right], \varphi\left(\left[f_{0}, \mathrm{smb}\right]\right)=\left[f_{0}, \mathrm{smb}\right]$. Рассмотрим $n$-листное накрытие $\left[f_{0}, \mathrm{smb}\right] \rightarrow\left[f_{0}, \mathrm{smb}\right] / \mathbb{Z}_{n}$. Проведя рассуждения, обобщающие рассуждения из [1] на наш случай, можно доказать гомеоморфность пространства $\left[f_{0}, \mathrm{smb}\right] / \mathbb{Z}_{n}$ стягиваемому многообразию. Из этого, помимо теоремы 2 , следует, что отображение $\varphi$ порождает циклическую перестановку компонент связности многообразия $\left[f_{0}, \mathrm{smb}\right]$.

Изложим схему доказательства теоремы 1 . Тот факт, что множество $\left[f_{0}\right]$ непусто, мы получаем в ходе доказательства теоремы 2. Далее, рассматривая комплексные формы полиномов, несложно убедиться в связности пространства $\left[f_{0}\right] / \mathbb{Z}_{n}$. Подчеркнем, что это верно и при $k=n$.

Следующий шаг заключается в построении пути в $\left[f_{0}\right]$, соединяющего $f$ и $\varphi f$ для некоторого $f \in\left[f_{0}\right]$. При $k=n$ такой путь построить невозможно [3]. При $k<n$ мы делаем следующее: рассмотрим множество $M$-полиномов (полиномов с $2 n$ критическими точками на окружности). Пользуясь конструкцией многогранника $M$-полиномов из [3], можно построить в явном виде путь на границе этого 
многогранника, из которого сколь угодно малым шевелением можно получить искомый путь в $\left[f_{0}\right]$.

Третье утверждение теоремы непосредственно следует из результатов [3]. Кроме того, в силу единственности скобочного символа при $k=n$ это утверждение вытекает из теоремы 2.

5. Автор выражает благодарность В. И. Арнольду, В. М. Закалюкину, П. Е. Пушкарю и Ю. В. Чеканову за многочисленные полезные советы и обсуждения.

\section{ЛИТЕРАТУРА}

1. Баранников C. А. Функц. анализ и его прил., 26, вып. 2, 10-17 (1992). 2. Шапиро Б. 3. Мат. заметки, 62, № 4, 635-640 (1997). 3. Arnold V. I. In: Arnold-Gelfand Mathematical Seminars, Boston, Birkhäuser, 1997, pp. 101-106.

Московский государственный университет им. М. В. Ломоносова

Поступило в редакцию Независимый московский университет e-mail: shadrin@mccme.ru 